\title{
Radical anions of nitrobenzothiazoles. II. EPR study of free radical anions and ion pairs
}

\author{
Francesco Ciminale \\ Dipartimento di Chimica, Università di Bari, via Orabona 4, 70126 Bari, Italy \\ E-mail: ciminale@chimica.uniba.it
}

Dedicated to Professor Giuseppe Bartoli on the occasion of his 65th birthday

\begin{abstract}
The paramagnetic species produced by reduction of the title compounds (NBTZ) with different reducing agents have been characterized by EPR spectroscopy. Radical ion pairs were observed upon reduction with alkali metals (Li, Na, K) in THF only from 5-, 6-, and 7-nitrobenzothiazole. The free radical anion of 7-NBTZ was obtained by reduction with $\mathrm{Na}$ in HMPA. Different tetraethylammonium ion pairs of the radical anion of 7-NBTZ were detected in HMPA when the reduction was performed by electrolysis at variable concentration of tetraethylammonium perchlorate as supporting electrolyte.
\end{abstract}

Keywords: EPR spectroscopy, radical anions, ion pairs, nitrobenzothiazoles, one electron reduction

\section{Introduction}

I have recently reported an EPR study of the radical anions produced from all five nitrobenzothiazole (NBTZ) isomers by different reductive methods. ${ }^{1}$ The chemical reduction by $t$-BuOK in DMSO or by glucose and $\mathrm{MeOK}$ in $\mathrm{MeOH}$ presented some difficulties in generating the radical anions 2-, 4-, and 6- $\mathrm{NBTZ}^{-}$, while the electrochemical reduction in DMSO proved to be of more general applicability affording smoothly the full series of $\mathrm{NBTZ}^{-}$. A likely drawback of the chemical reduction was the inevitable presence of nucleophilic species along with the known $^{2 \mathrm{a}-\mathrm{c}}$ nucleophilic reactivity at C-2 of 2-, 4-, and 6- NBTZ. Following the decrease in the observed nitrogen hyperfine splitting constant, $a_{\mathrm{N}}\left(\mathrm{NO}_{2}\right)$, within the radical anions obtained by electrolytic reduction in DMSO, it could be established that the delocalizing (electronwithdrawing) ability of each benzothiazolyl system increases in the order: benzothiazol-5-yl < benzothiazol-6-yl, benzothiazol-7-yl < benzothiazol-4-yl < benzothiazol-2-yl. The values of $a_{\mathrm{N}}$ $\left(\mathrm{NO}_{2}\right)$ measured in $\mathrm{MeOH}$ were 3.08 to $3.99 \mathrm{G}$ larger than those in DMSO, which is 
characteristic of hydrogen bonded nitroaromatic radical anions. ${ }^{3}$ Minor differences in $a_{\mathrm{N}}\left(\mathrm{NO}_{2}\right)$ were also registered in DMSO between radical anions generated by chemical (t-BuOK) or electrochemical method (with $\mathrm{Et}_{4} \mathrm{~N}^{+} \mathrm{ClO}_{4}{ }^{-} 0.1 \mathrm{M}$ as supporting electrolyte). This should be interpreted as due to some environmental perturbation, ${ }^{4}$ namely the counterion $\left(\mathrm{K}^{+}\right.$or $\left.\mathrm{Et}_{4} \mathrm{~N}^{+}\right)$ interactions affecting the charge and spin distribution between the nitrogen and oxygen atoms of the nitro group.

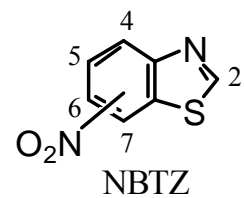

In the present work, free radical anions of NBTZs and ion pairs have been generated with appropriate reduction systems. The EPR characterization of the different paramagnetic species has been based on measurements of $a_{\mathrm{N}}\left(\mathrm{NO}_{2}\right)$ and, in the case of ion pairs, also of the hyperfine constant of the metal counterion, $a_{\mathrm{M}}$.

\section{Results and Discussion}

The reduction of nitrobenzothiazoles with alkali metals, namely $\mathrm{Li}$, $\mathrm{Na}$ and $\mathrm{K}$ in THF yielded paramagnetic species that could be easily characterized from their EPR spectra only in the cases of 5-, 6-, and 7- $\mathrm{NO}_{2}$ derivatives; for instance, the spectrum from 7-NBTZ is reported in Figure 1a. By contrast, reduction of 2- and 4-NBTZ yielded weak and poorly resolved resonances that were not interpreted, but at the same time served to rule out the formation of persistent radical anions of the parent nitrobenzothiazoles. Compared to the hyperfine patterns previously assigned to 5-, 6-, and 7- NBTZ - in DMSO, ${ }^{1}$ the spectral analysis in THF revealed two major differences: (a) an additional hyperfine coupling to metal nucleus $\left(a_{\mathrm{M}}\right)$, (b) larger values of $a_{\mathrm{N}}\left(\mathrm{NO}_{2}\right)$. The measured splitting constants, reported in Table 1, strongly indicate that 5-, 6-, and 7-NBTZ ${ }^{-}$are present in THF as contact ion pairs. The coupling constant $a_{\mathrm{M}}$, requiring that part of the spin density of the radical anion should be transferred to the diamagnetic counterion, is a direct evidence that radical ion and alkali metal are close one another forming a contact ion pair.

The magnitude of $a_{\mathrm{M}}$ is shown to decrease on going from sodium to potassium, becoming vanishingly small for the lithium counterion. This is the normal trend observed for the ion pairs of nitrobenzenides ${ }^{4-6}$ whose structure is characterized by having the metal located in the neighborhood of the oxygen atoms of the nitro group: the larger cation above the plane of the $\mathrm{NO}_{2}$ and midway between the oxygen atoms, the smaller one localized at either of the two oxygen atoms. ${ }^{5}$ According to this, the magnitude of $a_{\mathrm{M}}$ seems not to be linked to any definite physical quantity of the metal because it is the result of a balance between negative and positive spin densities created by different mechanisms of spin-transfer onto the diamagnetic cation. ${ }^{6}$ 
Table 1. Hyperfine coupling constants of ion pairs obtained by reduction of nitrobenzothiazoles with alkali metals in THF

\begin{tabular}{cccc}
\hline Nitrobenzothiazole & Alkali metal & $a_{\mathrm{N}}\left(\mathrm{NO}_{2}\right)$ & $a_{\mathrm{M}}$ \\
\hline $5-\mathrm{NBTZ}$ & $\mathrm{Li}$ & 11.32 & $-^{a}$ \\
& $\mathrm{Na}$ & 10.91 & 0.31 \\
& $\mathrm{~K}$ & 10.41 & 0.21 \\
6-NBTZ $^{b}$ & $\mathrm{Na}$ & 10.23 & 0.33 \\
& $\mathrm{~K}$ & 9.61 & 0.20 \\
$7-\mathrm{NBTZ}$ & $\mathrm{Li}$ & 10.47 & $c a .0 .09^{c}$ \\
& $\mathrm{Na}$ & 10.12 & 0.34 \\
& $\mathrm{~K}$ & 9.59 & 0.19 \\
\hline
\end{tabular}

${ }^{a}$ Unresolved. ${ }^{b}$ No paramagnetic resonance detected upon reduction with Li. ${ }^{c}$ Partially resolved by detection of the second harmonic of the EPR signal.

The significant rise in the values of $a_{\mathrm{N}}\left(\mathrm{NO}_{2}\right)$ in THF (Table 1), as compared to those in $\mathrm{DMSO},{ }^{1}$ is that expected for nitroaromatic radical anions, and reflects the polar interaction of the alkali metal and the nitro group. ${ }^{6}$ This is similar to, but less important than, the hydrogen bonding interaction in determining the shift of spin density from the nitrogen to the oxygen atoms. ${ }^{3}$ Also, the trend of $a_{\mathrm{N}}\left(\mathrm{NO}_{2}\right)$ with the nature of metal agrees with the fact that small cations are expected to perturb the spin distribution within the nitro group more strongly than large cations. ${ }^{6}$

Stevenson et al. generated the radical anion of nitrobenzene by reduction with alkali metals in HMPA, a solvent with powerful solvating ability for alkali metal ions, and detected ion pairs and free ions simultaneously. ${ }^{4}$ Moreover, they found that the residual tendency for nitrobenzenides to form ion pairs in this solvent decreases when electron-withdrawing groups are present in the para position. ${ }^{7}$

Applied to nitrobenzothiazoles, the reduction with $\mathrm{Na}$ in HMPA afforded the corresponding radical anion only in the case of 7-NBTZ. The formation of $7-\mathrm{NBTZ}^{-}$as free ion could be clearly inferred from its EPR spectrum shown in Figure 1b, particularly from the absence of metal splitting (compare with the spectrum of $7-\mathrm{NBTZ}^{-} \mathrm{Na}^{+}$ion pair in Figure 1a) and from a small value of $a_{\mathrm{N}}\left(\mathrm{NO}_{2}\right), 7.63 \mathrm{G}$. However, a closer inspection of the spectrum reveals that the free radical anion is very likely accompanied by a small amount of ion pair. The EPR signals that testify for the latter paramagnetic species would consist only of the set of very weak lines displayed on the left side of the spectrum - the expected hyperfine structure, lying symmetrically on the right side (high-field region), being lacking because of the line-broadening due to anisotropic effects on the nitrogen splitting. Concerning the consistency with the ion pair, one should note that the minor hyperfine structure (above) exhibits the characteristic metal splitting (quartet); the coupling constant is $0.3 \mathrm{G}$. On the other hand, the fact that little ion pairing is 
observed would reflect a decrease in the charge density of the $\mathrm{NO}_{2}$ group ${ }^{7}$ that agrees with the electron-withdrawing effect of the benzothiazol-7-yl system. ${ }^{1}$
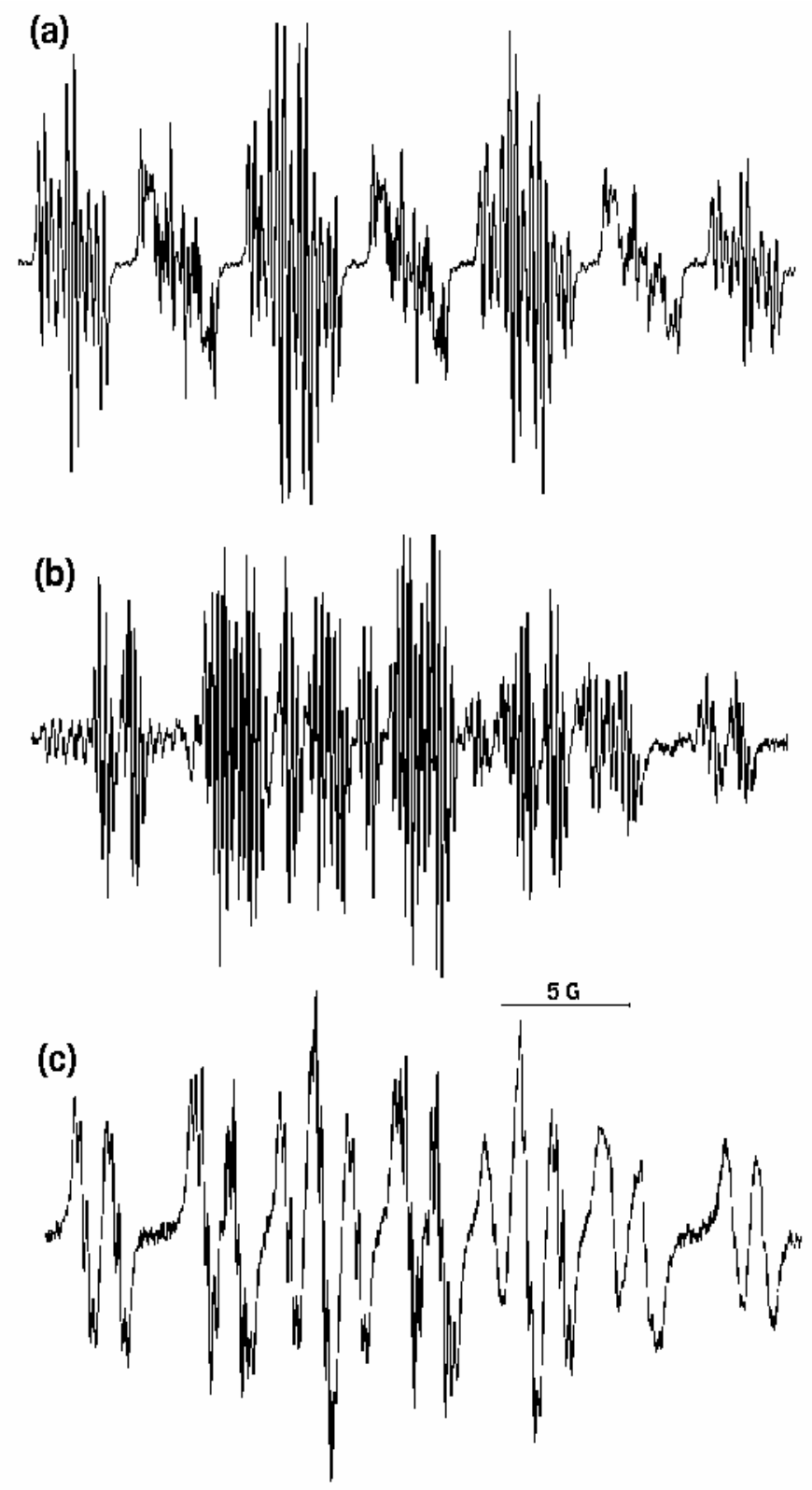

Figure 1. EPR spectra of 7-NBTZ ${ }^{-}$obtained upon sodium reduction (a) in THF and (b) in HMPA, and (c) by electrolysis with TEAP $0.1 M$ in HMPA.

The electrochemical reduction of 7-NBTZ in HMPA $\left(10^{-3} M\right)$ with tetraethylammonium perchlorate (TEAP, $0.1 \mathrm{M}$ ) as supporting electrolyte yielded a single radical anion, whose EPR spectrum is shown in Figure 1c. For $a_{\mathrm{N}}\left(\mathrm{NO}_{2}\right)$, a value of $8.05 \mathrm{G}$ was measured-that is $0.42 \mathrm{G}$ larger than the coupling constant observed for the free radical anion (Na reduction in HMPA), 
and $0.70 \mathrm{G}$ smaller than the value obtained for the electrochemical reduction in DMSO under identical experimental conditions. ${ }^{1}$ The first difference indicates that in HMPA the radical anion 7-NBTZ ${ }^{-}$is subject to interactions with the $\mathrm{Et}_{4} \mathrm{~N}^{+}$ion; in fact, this ion would be less solvated than $\mathrm{Na}^{+}$in HMPA. ${ }^{8}$ The second difference indicates that spin density on $\mathrm{NO}_{2}$ is perturbed by the $\mathrm{Et}_{4} \mathrm{~N}^{+}$cation more in DMSO than in HMPA. This would be the expected result if the greater affinity of HMPA for tertiary ammonium cations ${ }^{9}$ applies also to tetraalkylammonium cations.

As already reported for nitrobenzene, ${ }^{7}$ the magnitude of $a_{\mathrm{N}}\left(\mathrm{NO}_{2}\right)$ for 7-NBTZ ${ }^{-}$was found to decrease with decreasing concentration of TEAP. A plot of $a_{\mathrm{N}}\left(\mathrm{NO}_{2}\right)$ versus electrolyte concentration is shown in Figure 2. Curve-fitting of this plot allows us to calculate the value of $a_{\mathrm{N}}\left(\mathrm{NO}_{2}\right)$ at zero electrolyte concentration, when the radical anion is obviously free from the perturbing effect of $\mathrm{Et}_{4} \mathrm{~N}^{+}$ion. In fact, the value of $7.66 \mathrm{G}$, calculated for $a_{\mathrm{N}}\left(\mathrm{NO}_{2}\right)$ at zero TEAP concentration, is almost the same as that of free $7-\mathrm{NBTZ}^{-}$obtained upon reduction with $\mathrm{Na}$ in HMPA.

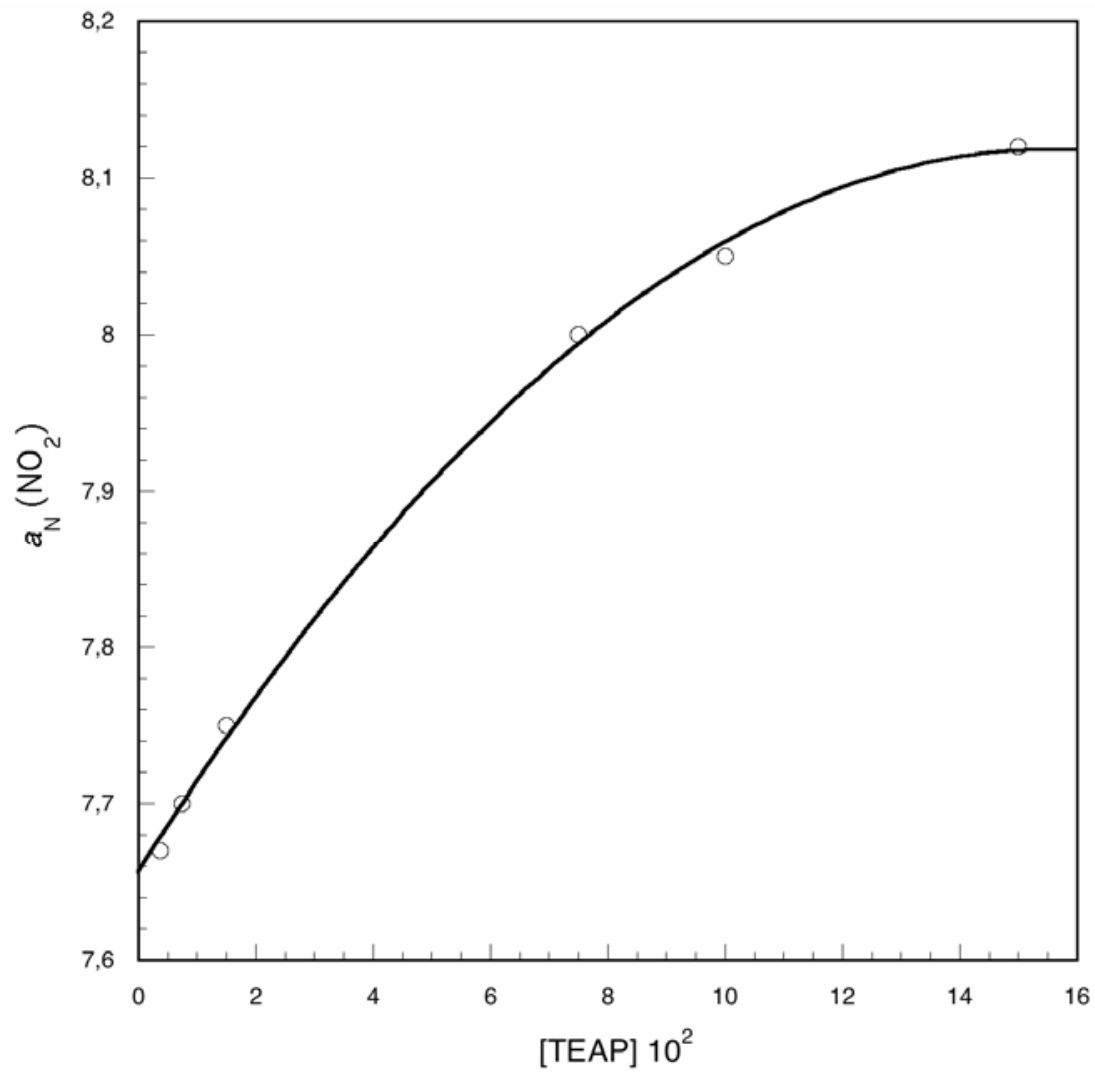

Figure 2. Plot of nitrogen $\left(\mathrm{NO}_{2}\right)$ splitting vs molar concentration of electrolyte for 7-NBTZ generated by electrolysis in HMPA.

Stevenson and his coworkers, considering the solvent perturbation to be negligible, have calculated the contribution of cation perturbation to $a_{\mathrm{N}}$ of alkali-metal-nitrobenzenide ion pairs 
in HMPA. ${ }^{4}$ The value they obtained for the ion pair of Na, by subtracting $a_{\mathrm{N}}$ of free ion from $a_{\mathrm{N}}$ of ion pair, is 2.5. According to this, it is interesting that also for $7-\mathrm{NBTZ}^{-} \mathrm{Na}^{+}$ion pair I find exactly the same contribution of cation perturbation to $a_{\mathrm{N}}\left(\mathrm{NO}_{2}\right)$, even though the coupling constants utilized for the calculation in this case are measured in different solvents: THF for the ion pair $\left[a_{\mathrm{N}}\left(\mathrm{NO}_{2}\right)=10.12 \mathrm{G}\right]$ and HMPA for the free ion $\left(a_{\mathrm{N}}\left(\mathrm{NO}_{2}\right)=7.63 \mathrm{G}\right)$.

\section{Experimental Section}

General Procedures. Nitrobenzothiazoles were available from a previous study. ${ }^{1}$ Tetrahydrofuran was refluxed over $\mathrm{LiAlH}_{4}$, distilled and stored over sodium wire under nitrogen. Hexamethylphosphoramide was refluxed over calcium hydride, distilled and stored over 4A molecular sieves. Tetraethylammonium perchlorate (Fluka) was used as supplied.

EPR measurements. The EPR spectra were recorded on a Varian E-109 spectrometer. The radical ion pairs, in THF, were produced in vacuum-sealed cells by contact of the substrate solution with a mirror of $\mathrm{Na}$ or $\mathrm{K}$, or with a clean piece of $\mathrm{Li}$. In the latter case, sonication in a cleaning bath was necessary to accomplish the process. The reduction with Na in HMPA was similar to that in THF. Electrochemical reductions of degassed HMPA solutions were carried out in an electrolytic flat cell at a cathode potential of $-0.9 \mathrm{~V} v \mathrm{~S}$ SCE; the concentration of the substrate was $\leq 10^{-3} \mathrm{M}$, while the concentration of the supporting electrolyte was varied within the range $0.0037-0.15 \mathrm{M}$.

\section{Acknowledgements}

This work was financially supported by Ministero dell'Istruzione, dell'Università e della Ricerca, Rome.

\section{References}

1. Ciminale, F. Tetrahedron Lett. 2004, 45, 5849.

2. (a) Todesco P. E. Bol. Sci. Fac. Chim. Ind. Bologna 1965, 23, 107. (b) Forlani L. J. Chem. Soc. Perkin Trans. 2 1991, 1699. (c) Bartoli, G.; Ciminale F.; Todesco, P. E. J. Chem. Soc. Perkin Trans. 2 1975, 1472.

3. Ludwig, P.; Layloff, T; Adams, R. N. J. Am. Chem. Soc. 1964, 86, 4568.

4. Stevenson, G. R.; Echegoyen, L.; Lizardi, L. R. J. Phys. Chem. 1972, 76, 1439.

5. Nakamura, K. Bull. Chem. Soc. Japan 1967, 40, 1 
6. Sharp, J. H.; Symons, M. C. R. In: Ions and Ions Pair in Organic Reactions; Swarc, M., Ed.; Wiley-Interscience, 1972; Vol. 1, pp. 185-218.

7. Stevenson, G. R.; Echegoyen, L. J. Phys. Chem. 1973, 77, 2339.

8. Stevenson, G. R.; Wilson, M. J. Phys. Chem. 1978, 82, 1171 and references therein.

9. Aitken, H. W.; Gikerson, W. R. J. Am. Chem. Soc. 1973, 95, 8551. 